$4 \quad$ Identification of Salivary Gland Escape Barriers to Western Equine Encephalitis

\author{
Virus in the Natural Vector, Culex tarsalis
}

6

7 Charles B. Stauft ${ }^{1 *}$, Aaron T. Phillips ${ }^{2}$, Tony T. Wang ${ }^{1}$, Kenneth E. Olson ${ }^{2}$

$11{ }^{1}$ Laboratory of Vector-Borne Diseases, Division of Viral Products, Office of Vaccine Research

12 and Review, Food and Drug Administration, White Oak, Maryland, United States of America

13 2Department of Microbiology, Immunology, and Pathology, College of Veterinary Medicine and

14 Biomedical Sciences, Colorado State University, Fort Collins, Colorado, United States of

\title{
15 America
}

16

17

18

* Corresponding author

19 E-mail: Charles.Stauft@fda.hhs.gov 


\section{Abstract}

Herein we describe a previously uninvestigated salivary gland escape barrier (SEB) in virus (WEEV). The WEEV strains were originally isolated either from mosquitoes (IMP181) or a human patient (McMillan). Both IMP181 and McMillan viruses were fully able to infect the salivary glands of Culex tarsalis after intrathoracic injection as determined by expression of mCherry fluorescent protein. IMP181, however, was better adapted to transmission as measured by virus titer in saliva as well as transmission rates in infected mosquitoes. We used chimeric recombinant WEEV strains to show that inclusion of IMP181-derived structural genes partially circumvents the SEB.

\section{Author Statement}

32 During the first half of the previous century, WEEV was responsible for large outbreaks throughout the northern United States and Canada that caused severe disease in horses and people. Over the past 60 years, cases of WEEV have mysteriously faded and the pathogen is rarely encountered in the clinic today. Salivary gland escape barriers (SEB) are a relatively neglected field of study in arbovirology, and this study provides a valuable contribution to the

37 field by describing a SEB found in otherwise vector competent Culex tarsalis mosquitoes. Although midgut barriers are well studied, less is known about barriers to transmission in the salivary glands. Although salivary gland infection occurs at a high rate following direct injection of virus into the hemocoel, we noticed that only $\sim 20-30 \%$ of infected mosquitoes transmit

41 detectable infectious virus in their saliva. Additionally, although the more pathogenic patient-

42 derived McMillan strain of WEEV infected salivary glands at a similar rate, its transmission was 
43 more severely restricted than the mosquito-derived but less pathogenic Imperial 181 strain. We

44 were able to trace determinants of viral transmission to the $6 \mathrm{~K} / \mathrm{E} 1 \mathrm{region}$ of the gene encoding

45 the viral structural polyprotein. WEEV is a valuable research model for the closely related

46 Eastern equine encephalitis virus and Venezuelan equine encephalitis virus we believe that our

47 findings are applicable to other members of Togaviridae.

\section{$48 \quad$ Introduction}

Western equine encephalitis virus (WEEV) is maintained in an enzootic transmission

50 cycle between Culex tarsalis and passerine bird species. Except for a few recorded incidences of

51 viremia in equine species (1-3), infection of humans or horses is considered a dead-end for viral

52 propagation (1). However, WEEV transmission to equine or human hosts has been associated

53 with severe disease outbreaks, and human survivors of encephalitic infection may experience

54 long-term neurological sequelae (4-7). More recently, WEEV McMillan has been used to model

55 viral-induced parkinsonism in a mouse model of disease (8). The molecular determinants of

56 WEEV McMillan neurovirulence in mice have been mapped to the structural protein E2 (9).

57 In the mosquito, natural infection occurs per os through the ingestion of a blood-meal

58 containing infectious virus. The virus is able to infect the midgut of the mosquito, replicate,

59 escape the midgut into the hemocoel, and then infect the salivary glands for a second round of

60 replication followed by release into the salivary gland lumen (10). Midgut entry and escape

61 barriers have previously been observed in WEEV infection of $C x$. tarsalis $(11,12)$ and a salivary

62 gland infection barrier has also been described (11). These previous studies have stopped short of

63 describing barriers to secretion of infectious virus particles into the saliva. High concentrations

64 of alphaviruses can be detected in mosquito saliva. Depending on the alphavirus and vector

65 species combination, concentrations from 1,000 to 100,000 mouse $\mathrm{LD}_{50}$ per mosquito were 

isolated from extracted mosquito saliva (18). Saliva titers of a highly related alphavirus, Highlands J virus, was reported to be $3.89 \times 10^{3} \mathrm{PFU} /$ saliva sample (19). However, the mechanisms of barriers to alphavirus transmission at the salivary gland level remain incompletely understood. molecular determinants for IMP181, in terms of mosquito midgut infectivity, exist in the same region of E2 as was identified for neurovirulence in mice. Although the mosquito infectivity and neurovirulence in mice have been reported for McMillan and IMP181 isolates in these earlier studies, there has not been an investigation of salivary gland barriers to transmission. In this report, we describe the existence of a salivary gland escape barrier for WEEV in CX. tarsalis and provide evidence for the role of the structural proteins in negotiating this barrier.

\section{Materials and methods} caught in Imperial County, CA and used to construct an infectious clone which was obtained from Dr. Aaron Brault at the Centers for Disease Control and Prevention. 
89 mCherry fluorescent protein was also introduced into the MCS downstream of the native 26S

90 sub-genomic promoter of the 5'dsWEEV.IMP181 plasmid. The primers used to amplify

91 mCherry gene inserts for cloning into pMcM-mCherry were 5'-

92 AAAACCGCGGATGGTGAGCAAGGG and AAAACCTGCAGGTTACTTGTACAGCTCG-

93 3'. The primers used to amplify mCherry gene inserts for cloning into pIMP181-mCherry were

94 5'-AAACCGCGGATGGTGAGCAAGGG-3' and 5'-AAACCCGGGTTACTTGTACAGCTCG-

95 3'.

Once amplified and sequenced, plasmids were linearized by incubation overnight with

97 MfeI (McMillan; New England Biolabs) or NotI (IMP181; New England Biolabs) at $37^{\circ} \mathrm{C}$.

98 Otherwise, generation of virus from the infectious clones was conducted as reported previously

99 (24). Infectious clones of the chimeric viruses were constructed previously (9) and are

100 composed of reciprocal crosses of C/E3/E2 or 6K/E1 regions from McMillan and IMP181 (Fig

101 1A).

102

Growth curves of the WEEV strains were conducted in C6/36 cells (ATCC) grown at $28^{\circ} \mathrm{C}, 5 \% \mathrm{CO}_{2}$ in MEM (Gibco) supplemented with 10\% FBS (Gibco). Confluent monolayers of each cell type were infected with a MOI of 0.01 for each virus and aliquots of supernatant taken every 12 hours for 48 or 60 hours. WEEV was quantified using replicate ten-fold serial dilutions in $90 \mu \mathrm{L}$ MEM 7\% FBS followed by addition of Vero cell (ATCC) suspensions and calculation 107 of $\mathrm{TCID}_{50} / \mathrm{mL}$ using the method of Reed and Muench (21).

109 Mosquito infection 
112 humidity with a 16:8 light:dark cycle prior to transfer to BSL-3 for WEEV infection at 1 week

113 post-emergence. In BSL-3, the mosquitoes were incubated at $28^{\circ} \mathrm{C}$ and $80.6 \%$ humidity in a

114 Caron 6030 environmental growth chamber. Cx. tarsalis were IT injected with 500, 250, or 125

115 TCID $_{50}$ of McMillan ( $\left.n=89\right)$, McM-mCherry $(n=73)$, IMP181 ( $\left.n=83\right)$, IMP181-mCherry $(n=73)$,

116 McM-IMP6K/E1 (n=78), IMP-McM6K/E1 (n=96), McM-IMPCE3E2 (n=96), or IMP-

117 McMCE3E2 (n=96). At 7 and 14 days post infection mosquitoes were induced to secrete saliva

118 by inserting the proboscis into a capillary tube containing $5 \mu \mathrm{L}$ of $50 \%$ FBS:glycerol solution

$119(15,18)$

Salivation proceeded for 30-45 minutes and the salivary glands were dissected for virus

121 detection. The proportion of $C x$. tarsalis transmitting virus was determined as the number of

122 saliva samples positive for infectious virus divided by the total number of saliva samples.

123 Infected salivary glands were identified by fluorescence microscopy or immune-fluorescence

124 assay (IFA) using an anti-SINV E1 antibody (30.11a). Any mosquitoes found to be negative for 125 salivary gland infection were excluded from further analysis.

126 Negative controls consisted of saliva collected from non-infected mosquitoes and diluted

127 in MEM with 7\% FBS. Positive controls included known doses of McMillan (5 x $10^{6}$ PFU)

128 strain WEEV added to saliva collected from non-infected mosquitoes either in the capillary tube 129 or after dilution in medium.

\section{Statistics}

At each time point, virus titers from end-point assays were compared using a Student's T-

132 test for data with normal distributions and Satterthwaite T-test for data lacking a normal

133 distribution. Infectious virus titers in saliva were log transformed and subjected to statistical

134 analysis using Student's t-test with a post hoc Bonferroni adjustment. The Bonferroni adjustment 
was used to counteract the loss of statistical power inherent in making multiple statistical comparisons and resulted in an alpha (and a significant p-value) of 0.01 . Graphs were assembled using GraphPad Prism (La Jolla, California).

Rates of transmission were compared between each set of related groups (14dpi versus 7dpi, McMillan versus IMP181, IMP181-mCherry versus IMP181, McM-mCherry versus McMillan, mosquitoes inoculated with 500 TCID $_{50}$ versus 250 TCID $_{50}$ and 125 TCID $_{50}$ infected mosquitoes) using confidence intervals of binomial proportions and Z-tests using statistical analysis software (SAS).

\section{RESULTS}

\section{Virus construction and growth in cell culture}

To develop an effective model to study the interactions of WEEV with its natural vector, double sub-genomic recombinant viruses expressing mCherry were constructed. The two principal strains of WEEV used in this study were isolated originally either from a human (McMillan) or Cx. tarsalis (IMP181) and propagated in mouse brains or Vero cells respectively an unknown number of times $(4,22,23)$ before being used to construct infectious clones $(24,25)$. McMillan and IMP181 have 99.7\% identity at the nucleotide sequence level and significant amino acid sequence identity. McMillan and IMP181 differ greatly in their ability to cause disease in an outbred CD-1 mouse model of infection. While both strains are neuroinvasive, IMP181 caused no mortality in mice after intranasal or subcutaneous infection but McMillan caused high mortality within 5 days (26). Enzootic strains such as IMP181 are associated with a lack of neurovirulence in mice (4). The McMillan strain was originally isolated in 1941 and has been used in numerous studies of WEEV (4,26-28). The growth kinetics for wildtype and 
infectious clone derived IMP181 and McMillan as well as chimeric recombinants and mCherry expressing constructs were compared in mosquito derived C6/36 cells (Fig 1B). All viruses demonstrated similar growth kinetics with a logarithmic increase in titers through the 48-hour time point (Fig 1B). Additionally, growth kinetics of McM-mCherry and IMP181-mCherry were compared to wild-type McMillan and IMP181 viruses. Peak titers were not significantly reduced in recombinant virus strains in C6/36 cells despite the addition of a second sub-genomic promoter and the gene encoding mCherry fluorescent protein.

Figure 1: Illustration of WEEV constructs and growth kinetics in vitro. A panel of infection. expressing recombinant viruses (McM-mCherry and IMP181-mCherry), we confirmed that the

177 salivary gland infection rate for McMillan and IMP181 compared to McM-mCherry or IMP181178 mCherry viruses were not significantly different 7 days following injection with 500 TCID $_{50}$ 179 (n=73-96, Fig 2). Additionally, McM-mCherry and IMP181-mCherry viruses displayed similar patterns of mCherry expression with uniform expression in the distal and proximal lateral lobes 
181

182

183

184

185

186

187

188

189

190

191

192

193

194

195

196

197

198

199

200

201

202

203

and the medial lobe of each set of salivary glands examined at 7 DPI (Fig 2B and 2C). In addition to mCherry expression, anti-SINV E1 antibody (30.11a) also revealed an almost complete infection of the salivary glands by both strains of virus at 7 DPI (Fig $2 \mathrm{~F}$ and $2 \mathrm{H})$. The 30.11a antibody also recognizes McMillan and IMP181 E1 due to the recombinant nature of WEEV. In salivary glands that were incompletely infected, the distal tips of the lateral lobes exhibited a lack of fluorescence. Prominent tissues expressing viral antigen included acinar cells and duct tissue connecting the glands to the proboscis.

Epifluorescent imaging of salivary glands was conducted to determine the presence or absence of a salivary gland infection barrier (SIB) in $C x$. tarsalis. Salivary gland infection by McM-mCherry or IMP181-mCherry would be marked by the presence or absence of mCherry expression. There were no visible differences in the pattern of salivary gland infection or frequency of infected salivary glands between IMP181-mCherry and McM-mCherry (Fig 2).

Salivary gland infection rates associated with each virus were not significantly different and ranged from $74 \%$ to $89 \%$, indicating the absence of a significant SIB. The rates of salivary gland infection were not significantly different between different doses $\left(125,250\right.$, and $\left.500 \mathrm{TCID}_{50}\right)$ of McM-mCherry and IMP181-mCherry at 7 days (Fig 2I) or 14 days post infection (Fig 2J).

\section{Figure 2: Epifluorescent imaging of $C x$. tarsalis salivary glands and rates of salivary gland}

infection. Salivary glands were compared to negative control epifluorescent (A) and light (D) images of non-infected $C x$. tarsalis salivary glands at 20x magnification. $C x$. tarsalis salivary glands 7 days post intrathoracic injection with 500 TCID $_{50}$ McM-mCherry (B) and IMP181mCherry (C). IFA of $C x$. tarsalis salivary glands. $C x$. tarsalis salivary glands stained immunohistochemically for WEEV 7 days post intrathoracic injection with $500 \mathrm{TCID}_{50}$ 
McMillan (F) and IMP181 (H) along with light microscopy images of the same salivary glands (E and G). Salivary gland infection rates were calculated using the number of fluorescent mosquitoes compared to total injected mosquitoes at 7 (I) and 14 (J) DPI. DL, distal-lateral lobe; M, medial lobe; PL, proximal-lateral lobe. DPI: Days post-infection. Reporter expressing recombinant viruses (IMP181-mCherry and McM-mCherry) were used to assess the impact of varied dosage and duration of infection on transmission rates as measured by the percentage of saliva containing detectable infectious virus. Rates of transmission were not found to vary significantly within groups of $C x$. tarsalis injected with different doses of IMP181-mCherry. However, McM-mCherry infected mosquitoes showed a significant increase in transmission rate from doses of 250 to 500 TCID50 at 7 and 14 days post injection (Fig 3A and 3B). Transmission rates were significantly greater for IMP181-mCherry than McM-mCherry (Fig 3A) at 7 days post injection with 125, 250, and $500 \mathrm{TCID}_{50}$ (Fig 3). After 14 DPI, transmission rates were found to be higher in IMP181-mCherry compared to McM-mCherry infected mosquitoes at the lowest dose of 125 TCID50, however, transmission rates were similar at the 250 and 500 TCID50 doses. The transmission rate was found to be dose dependent with McM-mCherry, however, no dose-dependence was observed in the dose range attempted for IMP181-mCherry infections (Fig 3A and 3B).

Despite similarity in rates of salivary gland infection, significant differences were observed in the concentration of infectious WEEV recovered in mosquito saliva (Fig 3). IMP181 infected mosquitoes secreted significantly more virus in their saliva compared to McMillan injected mosquitoes at 7 DPI ( $p=0.0011$; Student's T test). Following up on this difference, saliva virus titers were measured for chimeric McM-IMP181 recombinant viruses. IMPMcM6K/E1 (p=0.0010) and IMP-McMC/E3/E2 ( $\mathrm{p}=0.0066)$ had higher infectious virus titers in 
227 saliva compared to wild-type McMillan at 7 days post injection. IMP-McM6K/E1 and IMP-

$228 \mathrm{McMC} / \mathrm{E} 3 / \mathrm{E} 2$ also demonstrated significantly different saliva titers when compared to IMP181

$229(p=0.2602$ IMP-McM6K/E1; 0.1706 IMP-McMC/E3/E2; Fig 3C). Despite differences in the

230 concentration of expectorated virus, transmission rates were broadly similar between groups

231 (Figure 3D). Although approaching significance in a one-tailed test comparing binomials

$232(\mathrm{p}=0.0504)$, IMP181 transmission rates were not found to be higher than those in McMillan

233 infected mosquitoes. However, a significant difference was observed in IMP-McM6K/E1

234 infected mosquitoes compared to the McMillan group (Fig 3D). In these chimeric recombinant

235 viruses, increases in salivary gland titers associated with the IMP181 structural genes for $6 \mathrm{~K}$ and

236 E1. We did not observe significantly different concentrations of infectious virus in the saliva of

$237 \mathrm{McM}-\mathrm{IMPC} / \mathrm{E} 3 / \mathrm{E} 2$ or IMP-McM6K/E1, which possesses the nonstructural and 6K/E1 regions of

238 McMillan. A protein BLAST comparing the McMillan (ACT75276) and IMP181 (ACT75278)

239 structural polyproteins reveals 32/1236 amino acid mismatches. Of these mismatches 22 are in

240 the $\mathrm{C} / \mathrm{E} 3 / \mathrm{E} 2$ region and 10 are in $6 \mathrm{~K} / \mathrm{E} 1$. In future studies, further subcloning and site-directed

241 mutagenesis may enable the location of key mutations involved in egress from infected salivary

242 glands.

244 Figure 3: Salivary gland infection rates and infectious virus concentration in saliva.

245 Salivary gland infection rates at 7 (A) and 14 (B) days were compared between IMP181-

246 mCherry (dark grey) and McMillan (light grey) infected mosquitoes injected with 125, 250, or

$247500 \mathrm{TCID}_{50}$. Infectious viral titers were also compared between IMP181 and McMillan derived

248 viruses in saliva collected at 7 days post-injection with 500 TCID50 with IMP181 6K/E1 genes 
249

250

251

252

253

254

255

256

257

258

259

260

261

262

263

264

265

266

267

268

269

270

271

associated with an increase in expectorated virus (C). Transmission rates were also assessed for IMP181 and McMillan derived viruses (D).

\section{Conclusions}

A salivary gland infection barrier (SIB) was not detected with the Culex tarsalis CA strain for McMillan or IMP181 after intrathoracic injection as salivary gland infection was not shown to vary by dose, time, or strain of virus in this study. This observation agrees with previous work $(\mathbf{1 1}, \mathbf{1 2})$. Both McM-mCherry and IMP181-mCherry could infect $C x$. tarsalis salivary glands and express a fluorescent reporter that allowed for clear demarcation of infected versus uninfected tissue that was comparable to IFA. Dose dependence of transmission suggested a relationship between virus titer in the hemocoel to the expectoration of virus into the saliva of infected mosquitoes. Time after infection ( 7 or 14 days post intrathoracic injection) was not shown to significantly affect salivary gland infection, transmission rate in saliva, or amount of WEEV expectorated.

Previous work (29) identified a SIB in a different strain of $C x$. tarsalis. Our work identified a salivary gland escape barrier to WEEV in the CA strain of $C x$. tarsalis. An escape barrier was likely responsible for the lack of virus secreted in the saliva of infected mosquitoes as rates of transmission varied between $14-30 \%$ while salivary gland infection averaged around 74$89 \%$ for both strains of WEEV. The amount of virus detected in saliva was significantly greater for IMP181, clone 40, and clone 42 virus infected mosquitoes. IMP181-derived structural genes appeared to have an effect with regards to the phenotype of increased expectoration of virus in saliva as transmission of McMillan was enhanced by the structural genes of IMP181. Both halves of the structural gene encoding region were equally important to replication or egress in 
272 the salivary gland tissue of Culex tarsalis. In agreement with prior work (9), the IMP181 strain

273 appears to be more highly adapted to replication in the mosquito vector than the highly passaged

274 clinical isolate strain McMillan. The McMillan strain likely has adapted to vertebrate cells after

275 lengthy passaging under laboratory conditions and therefore is a useful counterpoint for studying

276 wild-type isolates like IMP181 which have maintained the ability to infect and be transmitted by

277 competent vectors. IMP181 and McMillan form a dyad in the spectrum between vector

278 infectivity and vertebrate host virulence that have been used in past studies to yield useful

279 conclusions.

IFA revealed the presence of WEEV E1 along the walls of the salivary gland duct for

282 separate the roles of budding and encapsidation in determining the amount of WEEV egress in

283 this study. Infectious virus titer in the saliva did not increase significantly with dose of IMP181-

284 mCherry or McM-mCherry injected into the mosquito. This indicated that the limiting step for

285 WEEV egress occurred within the salivary gland as the amount of WEEV in the hemocoel did

286 not alter the amount of expectorated virus. Future studies using IFA with antibodies specific for

287 different WEEV structural proteins and confocal microscopy could contribute enhanced

288 visualization to the study of viral escape from infected salivary glands.

Transmission rate was shown to be related to inoculation dosage in McM-mCherry as rates were significantly higher in 500 TCID $_{50}$ injected mosquitoes. Dose dependence was not

291 observed with the transmission rate of IMP181-mCherry. Homogenous presentation of IMP181-

292 mCherry transmission was possibly due to injection doses being higher than the minimum

293 threshold for salivary gland infection, replication, or escape. Also, Cx. tarsalis has been shown to

294 be more susceptible to IMP181 compared to McMillan in terms of midgut infection (9). The 5' 
(AvrII to KpnI) or 3' (KpnI to terminus) sections of the structural polyprotein of IMP181

previously rescued mosquito infection with either McMillan or IMP181 backgrounds (9). The

\section{Acknowledgements}

We would like to thank Irma Sanchez-Vargas for assistance in dissecting mosquito salivary glands.

\section{Bibliography}

1. Bergren NA, Auguste AJ, Forrester NL, Negi SS, Braun WA, Weaver SC. Western equine encephalitis virus: evolutionary analysis of a declining alphavirus based on complete genome sequences. J Virol. 2014 Aug;88(16):9260-7.

2. Byrne RJ, French GR, Yancey FS, Gochenour WS, Russell PK, Ramsburg HH, et al. CLINICAL AND IMMUNOLOGIC INTERRELATIONSHIP AMONG VENEZUELAN, EASTERN, AND WESTERN EQUINE ENCEPHALOMYELITIS VIRUSES IN BURROS. Am J Vet Res. 1964 Jan;25:24-31.

3. Sponseller ML, Binn LN, Wooding WL, Yager RH. Field strains of western encephalitis virus in ponies: virologic, clinical, and pathologic observations. Am J Vet Res. 1966 Nov;27(121):1591-8. 
4. Bianchi TI, Aviles G, Monath TP, Sabattini MS. Western equine encephalomyelitis: virulence markers and their epidemiologic significance. Am J Trop Med Hyg. 1993 Sep;49(3):322-8.

5. Mulder DW, Parrott M, Thaler M. Sequelae of Western Equine Encephalitis. Neurology. 1951 Jul 1;1(7-8):318-318.

6. Palmer RJ, Finley KH. Sequelae of encephalitis; report of a study after the California epidemic. Calif Med. 1956 Feb;84(2):98-100.

7. Schultz DR, Barthal JS, Garrett G. Western equine encephalitis with rapid onset of parkinsonism. Neurology. 1977 Nov;27(11):1095-6.

8. Bantle CM, Phillips AT, Smeyne RJ, Rocha SM, Olson KE, Tjalkens RB. Infection with mosquito-borne alphavirus induces selective loss of dopaminergic neurons, neuroinflammation and widespread protein aggregation. NPJ Park Dis. 2019;5:20.

9. Mossel EC, Ledermann JP, Phillips AT, Borland EM, Powers AM, Olson KE. Molecular Determinants of Mouse Neurovirulence and Mosquito Infection for Western Equine Encephalitis Virus. PLOS ONE. 2013 Mar 27;8(3):e60427.

10. Myles KM, Pierro DJ, Olson KE. Comparison of the transmission potential of two genetically distinct Sindbis viruses after oral infection of Aedes aegypti (Diptera: Culicidae). J Med Entomol. 2004 Jan;41(1):95-106.

11. Kramer LD, Hardy JL, Presser SB, Houk EJ. Dissemination barriers for western equine encephalomyelitis virus in Culex tarsalis infected after ingestion of low viral doses. Am J Trop Med Hyg. 1981 Jan;30(1):190-7.

12. Oviedo MVN, Romoser WS, James CB, Mahmood F, Reisen WK. Infection dynamics of western equine encephalomyelitis virus (Togaviridae: Alphavirus) in four strains of Culex tarsalis (Diptera: Culicidae): an immunocytochemical study. Res Rep Trop Med. 2011 Apr 18;2011(2):65-77.

13. Collins WE. Studies on the transmission of Semliki Forest virus by anopheline mosquitoes. Am J Hyg. 1963 Jan;77:109-13.

14. Devine TL, Venard CE, Myser WC. Measurement of salivation by Aedes aegypti (L.) feeding on a living host. J Insect Physiol. 1965 Mar 1;11(3):347-53.

15. Hurlbut HS. Mosquito salivation and virus transmission. Am J Trop Med Hyg. 1966 Nov;15(6):989-93.

16. Lamotte LC. Japanese B encephalitis virus in the organs of infected mosquitoes. Am J Hyg. 1960 Jul;72:73-87. 
17. Thomas LA. DISTRIBUTION OF THE VIRUS OF WESTERN EQUINE ENCEPHALOMYELITIS IN THE MOSQUITO VECTOR, CULEX TARSALIS. Am J Hyg. 1963 Sep;78:150-65.

18. Smith DR, Carrara A-S, Aguilar PV, Weaver SC. Evaluation of Methods to Assess Transmission Potential of Venezuelan Equine Encephalitis Virus by Mosquitoes and Estimation of Mosquito Saliva Titers. Am J Trop Med Hyg. 2005 Jul 1;73(1):33-9.

19. Borland EM, Ledermann JP, Powers AM. Culex Tarsalis Mosquitoes as Vectors of Highlands J Virus. Vector Borne Zoonotic Dis Larchmt N. 2016 Aug;16(8):558-65.

20. Kenney JL, Adams AP, Weaver SC. Transmission Potential of Two Chimeric Western Equine Encephalitis Vaccine Candidates in Culex tarsalis. Am J Trop Med Hyg. 2010 Feb $1 ; 82(2): 354-9$.

21. Reed LJ, Muench H. A Simple Method of Estimating Fifty Per Cent Endpoints,. Am J Epidemiol. 1938 May 1;27(3):493-7.

22. Reisen WK, Fang Y, Brault AC. Limited Interdecadal Variation in Mosquito (Diptera: Culicidae) and Avian Host Competence for Western Equine Encephalomyelitis Virus (Togaviridae: Alphavirus). Am J Trop Med Hyg. 2008 Apr 1;78(4):681-6.

23. Zhang M, Fang Y, Brault AC, Reisen WK. Variation in Western Equine Encephalomyelitis Viral Strain Growth in Mammalian, Avian, and Mosquito Cells Fails to Explain Temporal Changes in Enzootic and Epidemic Activity in California. Vector-Borne Zoonotic Dis. 2011 Mar 1;11(3):269-75.

24. Phillips AT, Stauft CB, Aboellail TA, Toth AM, Jarvis DL, Powers AM, et al. Bioluminescent imaging and histopathologic characterization of WEEV neuroinvasion in outbred CD-1 mice. PloS One. 2013;8(1):e53462.

25. Phillips AT, Rico AB, Stauft CB, Hammond SL, Aboellail TA, Tjalkens RB, et al. Entry Sites of Venezuelan and Western Equine Encephalitis Viruses in the Mouse Central Nervous System following Peripheral Infection. J Virol. 2016 Jun 15;90(12):5785-96.

26. Logue CH, Bosio CF, Welte T, Keene KM, Ledermann JP, Phillips A, et al. Virulence variation among isolates of western equine encephalitis virus in an outbred mouse model. $\mathrm{J}$ Gen Virol. 2009;90(8):1848-58.

27. Hayes CG. Variations in biological properties of geographic strains of western equine encephalomyelitis virus before and after passage in Culex tarsalis and Culiseta melanura. Acta Virol. 1978 1978;22(5):401-9.

28. Trent DW, Grant JA. A Comparison of New World Alphaviruses in the Western Equine Encephalomyelitis Complex by Immunochemical and Oligonucleotide Fingerprint Techniques. J Gen Virol. 1980;47(2):261-82. 
bioRxiv preprint doi: https://doi.org/10.1101/2022.01.11.475797; this version posted January 11, 2022. The copyright holder for this preprint

(which was not certified by peer review) is the author/funder. This article is a US Government work. It is not subject to copyright under 17 USC 105 and is also made available for use under a CCO license.

389

390

391

392
29. Kramer LD, Hardy JL, Presser SB. Characterization of Modulation of Western Equine Encephalomyelitis Virus by Culex tarsalis (Diptera: Culicidae) Maintained at $32^{\circ} \mathrm{C}$ Following Parenteral Infection. J Med Entomol. 1998 May 1;35(3):289-95. 
(which was not certified by peer review) is the author/funder. This article is a US Government work. It is not subject to copyright under 17 USC

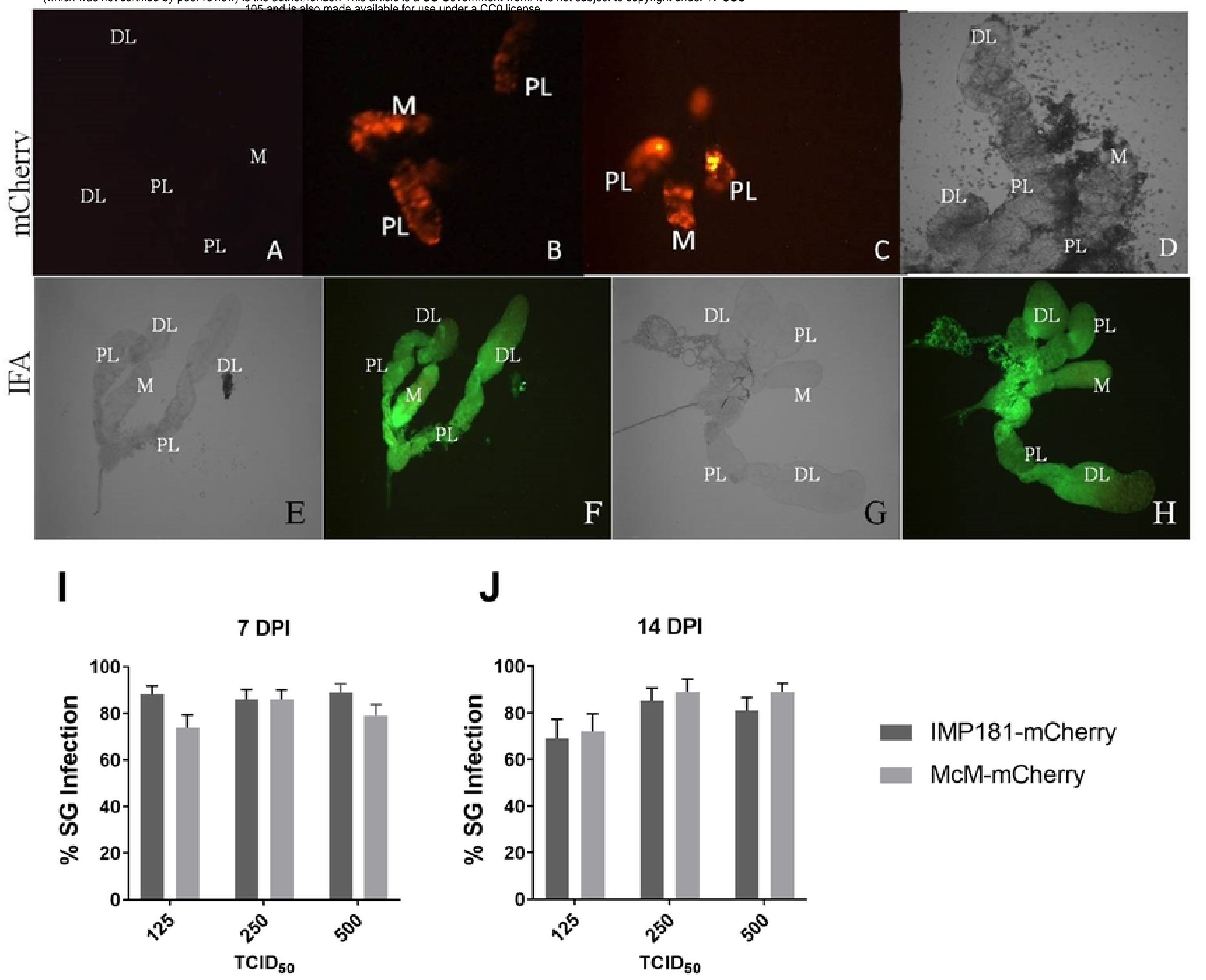

Figure 2 
A
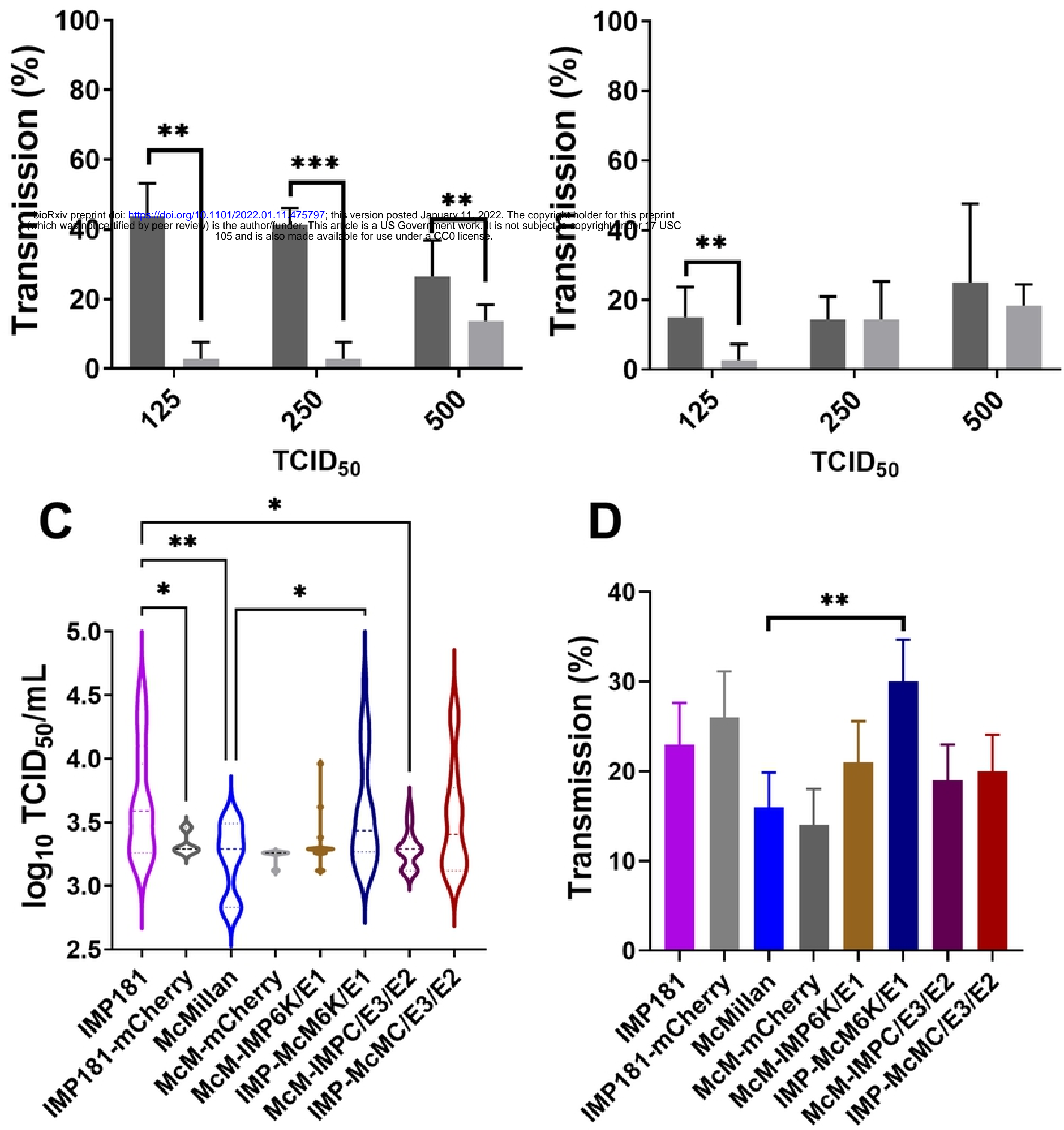

Figure 3 
A

\section{nsP1 nsP2 nsP3 nsP4 C E3 E2 6K E1}

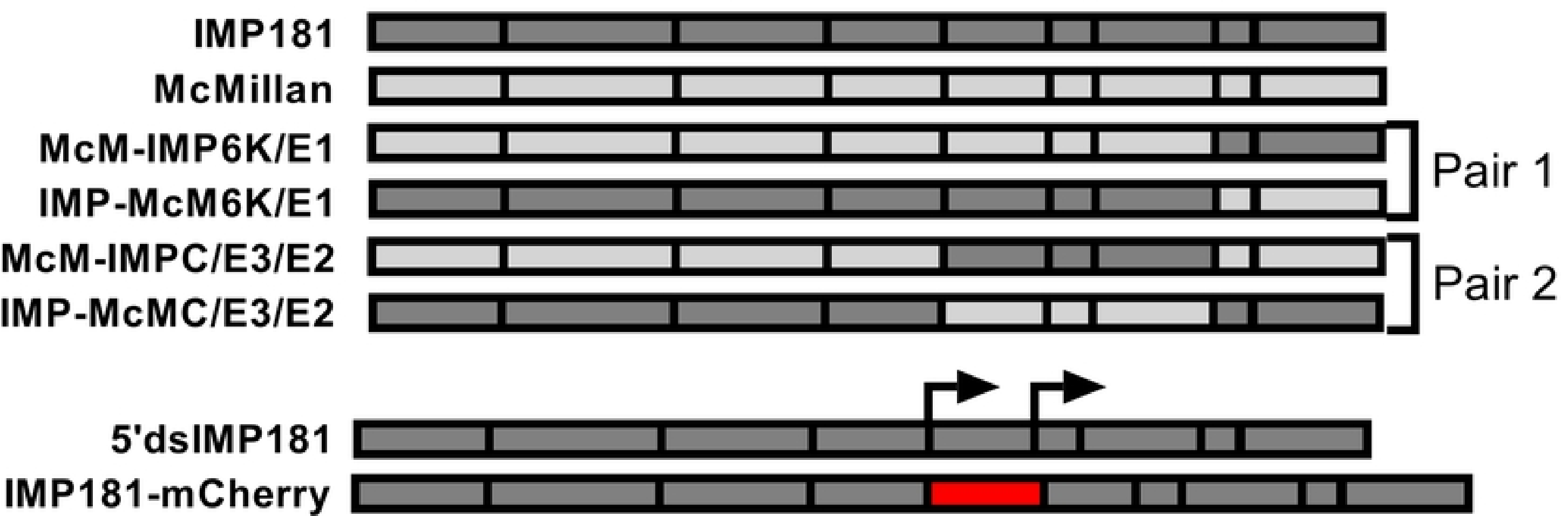

bioRxiv preprint doi: https://doi.org/10.1101/2022.01.11.475797; this version posted January 11, 2022. The copyright holder for this preprint

5'dsMcMillan

McM-mCherry

B

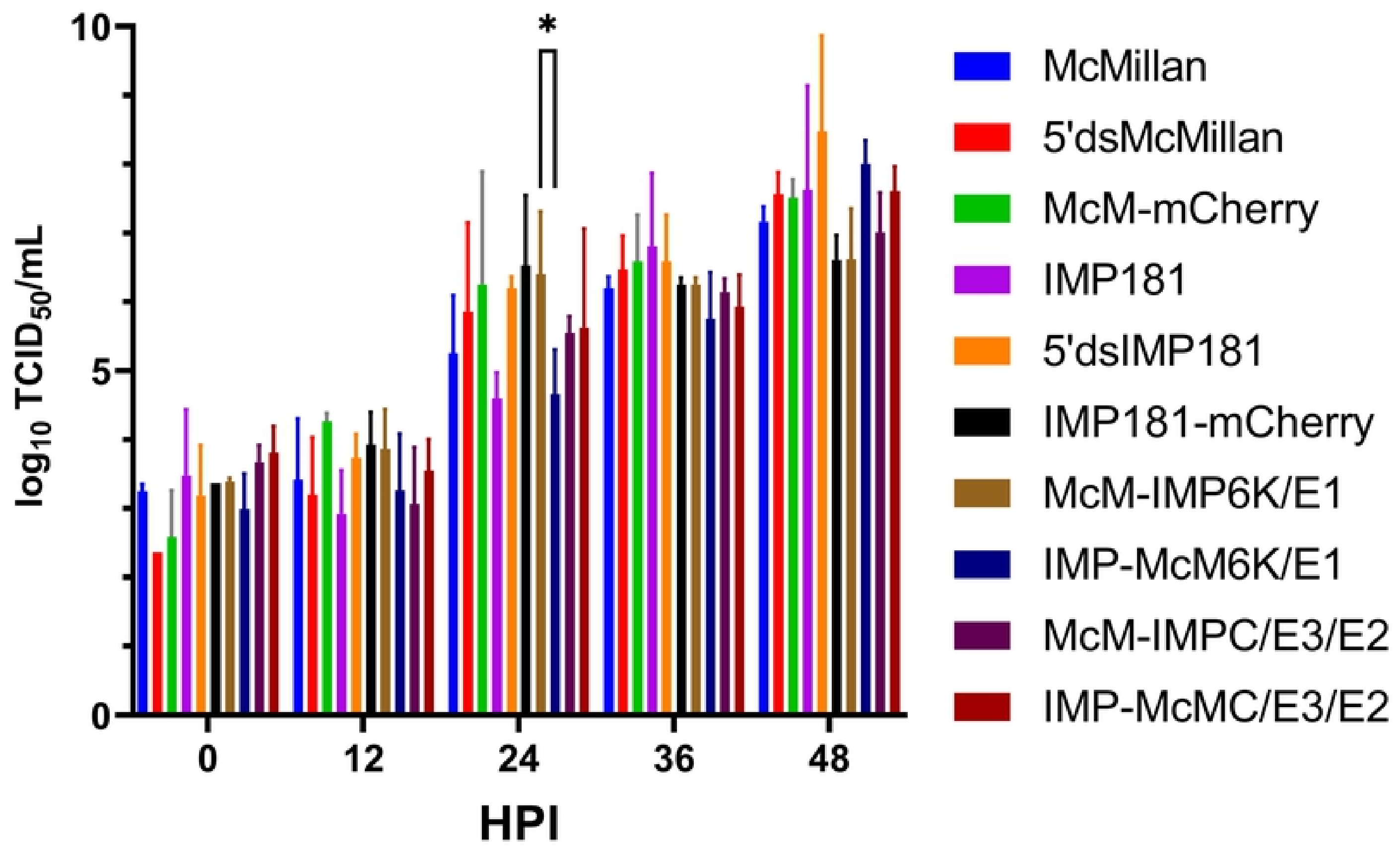

Figure 1 